\title{
LES NEMATODES SEURATOIDEA
}

\section{Par Alain G. CHABAUD, Yvonne CAMPANA-ROUGET et Edouard R. BRYGoo}

\section{Introduction}

Dans une note préliminaire (1959), nous avons proposé de rassembler dans une nouvelle super-famille d'Ascaridina: les Seuratoidea, un certain nombre de genres dispersés dans des familles variées et qui ont pour point commun une morphologie intermédiaire entre celle des Cosmocercoidea et celle des Spirurida.

L'objet du présent travail est de chercher à justifier les propositions taxonomiques qui ont été faites, en essayant de montrer que la super-famille semble n'être pas seulement le rassemblement de genres divers difficiles à classer, mais encore un ensemble qui, sous un aspect très varié, paraît avoir une homogénéité naturelle. Nous compléterons en même temps les définitions abrégées qui ont été proposées précédemment et ferons quelques additions au cadre primitivement proposé.

\section{Historique sommaire}

Les genres qui nous intéressent figurent actuellement dans des groupes variés et il est intéressant de voir quelles sont les raisons qui ont conduit à les y placer.

\section{a) CUCULLANIDE :}

Seurat (1915) donne la première description moderne du groupe avec Ophiostomum tacapense. Cette description est excellente, sauf sur un point, car la bouche est décrite et figurée comme ayant deux lèvres. L'auteur dit: "Le genre Ophiostomum, par la conformation des ailes caudales et la disposition des papilles génitales du mâle, par la forme et la dimension des œufs, se rattache aux Hétérakidés à deux lèvres buccales. Il diffère de ceux-ci par la conformation de l'œsophage, privé d'un bulbe distinct, et par l'absence 
de la ventouse préanale chez le mâle. Les Ophiostomum montrent, d'autre part, des affinités avec le genre Dichelyne Jägersk., qui appartient à la famille des Acuariidx. »

Hall (1916) constate que le nom d'Ophiostomum ne peut être employé et propose le nom de Seuratum. Il en fait le type d'une nouvelle sous-famille, Seuratinæ, avec la définition : «Heterakidæ : Transitional forms in which the male is without an anal sucker. Mouth with two lips. No œsophageal bulb present. Male with short, equal, or subequal spicules, and with a gubernaculum. Vulva near middle of body. Uteri divergent. »

La sous-famille est élevée au rang de famille par Railliet (1916). Baylis (1923) et Baylis et Daubney (1926), se basant évidemment sur le caractère de la bouche à deux lèvres, placent le genre dans les Cucullanidæ en indiquant toutefois :

«Hall (1916) has suggested a subfamily Seuratinæ for the reception of Seuratum. He considers that this subfamily should be included in the Heteralidx, although admitting that affinities of the genus are perhaps closer with Dacnitis, now recognised as a synonym of Cucullanus. It differs from Cucullanus in the absence of a sucker-like organ in the male, and in the absence of a marked dilatation of the anterior end of the œsophagus. It is, however, insufficiently described.

Yorke et Maplestone (1926) classent simplement la famille parmi les Spiruroidea.

Chitwood et Wehr reprennent les vues de Baylis et placent les Seuratinæ comme sous-famille des Cucullanidæ, point de vue qui est conservé dans tous les travaux récents. Bien que de nombreux auteurs aient étudié des Seuratum d'espèces variées, à bouche triangulaire, l'erreur de Seurat est constamment recopiée, et tout le monde semble avoir perdu de vue les affinités possibles avec les «Hétérakidés \&ui avaient cependant été admises primitivement.

Autour de Seuratum ont été placés différents genres et familles. Chitwood et Wehr (1934) y placent le genre Omeia Hsü 1933, le genre Haplonema Ward et Magath 1917, ainsi que le genre Pingus Hsü 1933, qu'ils considèrent comme synonyme du précédent (la famille des Pingidæ est donc placée en synonymie).

Paraseuratum Johnston et Mawson 1940, ainsi que Seuratinema Johnston et Mawson 1941a ont été classés par les auteurs euxmêmes près de Seuratum. Dollfus et Chabaud (1957) ont rapproché de Seuratinema le genre Skrjabinura Gnedina 1933, plaçant ainsi la famille Skrjabinuridæ en synonymie d'une partie de celle des Cucullanidx, et Thomas-Mawson (1960) a montré la synonymie de Seuratinema avec Skrjabinura. 
Enfin, Sudarikov et Ryjikov (1952) insistent sur les affinités de Cottocomephoronema et d'Ichtyobronema avec Haplonema.

b) QUIMPERIIDE :

La première description moderne d'une espèce est celle de Gendre (1926), qui crée le genre Quimperia. En 1928, il l'élève au niveau de sous-famille.

Baylis décrit en 1930 Gendria et le rapproche du précédent dans la famille des Quimperiidæ. Il précise que la famille ne peut être placée dans les Heterakidæ, comme l'avait proposé Gendre, mais plutôt à côté des Kathlaniidær. Il envisage également certaines affinités avec les Cucullanidæ.

En 1934, Baylis reprend une espèce de Linstow et fait un troisième genre, Paraquimperia; puis, en 1939, un quatrième genre, Paragendria, à partir d'une espèce décrite comme Hétérakide par Stewart (1914). Il redonne à cette occasion une définition de la famille.

Enfin, en 1954, Ali et Singh ajoutent le cinquième genre, Buckleynema.

Chitwood et Chitwood (1950) considèrent le groupe comme une sous-famille d'Heterakidæx, qu'ils placent après les Subulurinæ, en indiquant toutefois : " There seems to be some doubt as to whether this subfamily should be placed with the Heterakidx or the Cucullanidx. 》

Chabaud (1957) conserve le rapprochement avec les Subuluridæ, mais les place avant cette famille.

c) THELAZIIDE :

D'autres genres nous intéressent également, bien qu'ils aient toujours, jusqu'à maintenant, été considérés comme des Thelaziidæ. Ce sont le genre Skrjabinelazia, qui est actuellement bien décrit, et deux genres connus seulement de façon très partielle, Rictularina Johnston et Mawson 1941b, et Denticulospirura Johnston et Mawson $1941 c$.

d) HETERAKIDE :

L'Ascaris retusa Rud. 1819, après avoir été considéré comme un Heterakis, fut pris comme type d'un genre particulier : Schneidernema, puis d'une famille particulière. Inglis, en 1958, en rapproche le nouveau genre Morgascaridia (pour une espèce considérée également à l'origine comme un Hétérakide). Enfin, tout récemment, Inglis et Chabaud (1960) proposent le rattachement aux Seuratoidea. 


\section{Analyse des différents genres}

A) Genres classés habituellement parmi les Spirurides:

- Genre Seuratum (fig. 1 et 2).

Bouche: Nous avons pu nous assurer tout d'abord que les spécimens types de l'espèce type Seuratum tacapense n'ont pas la bouche bilabiée décrite primitivement, mais bien une bouche triangulaire (fig. $3 \mathrm{~A}$ ), comparable à celle des autres espèces du genre. La notion de symétrie bilatérale de la tête, qui a été certainement

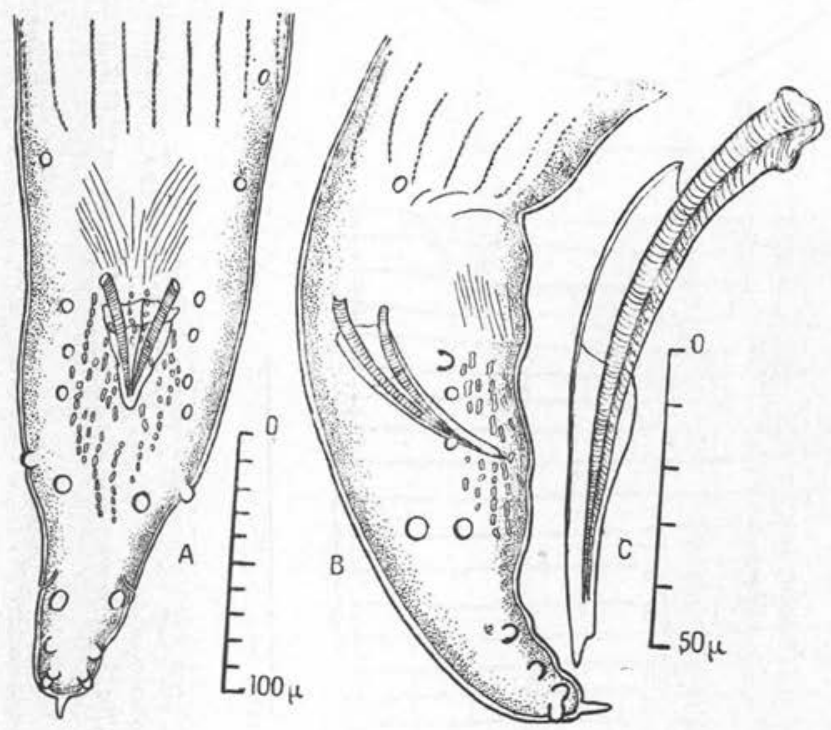

FIG. 2. - Seuratum mucronatum (Rud. 1809). Mâle.

A : Extrémitce postérieure; vue ventrale ; B : Extrémité postérieure ; vue latérale; $\mathrm{C}$ : Spicules et gubernaculum. D’après Biocea et Chabaud 1951.

l'élément essentiel dans la décision de Baylis, ne repose donc que sur une erreur. Une bouche triangulaire est tout à fait anormale pour un Spiruride.

Papilles céphaliques : Les huit papilles sont groupées 2 à 2 et la médio-médiane n'est pas nettement plus petite ou plus antérieure que la latéro-médiane correspondante. Cette disposition se voit plus facilement chez les Ascaridina, mais existe cependant chez certains Spirurida tels que les Dracunculides. 
Esophage : L'œsophage, court et cylindrique, est un caractère d'Ascaridina et non de Spirurida. Il n'y a que les Cucullanidæ qui aient cet œsophage cylindrique, et c'est pourquoi il a fallu mettre Seuratum dans cette famille, alors qu'il n'y a aucun élément commun dans la structure céphalique des deux groupes.

Musculature (fig. 4) : Il est impossible de faire une limite précise entre les musculatures "méromyaires » et les musculatures "polymyaires », car le passage de l'un à l'autre type se fait de façon

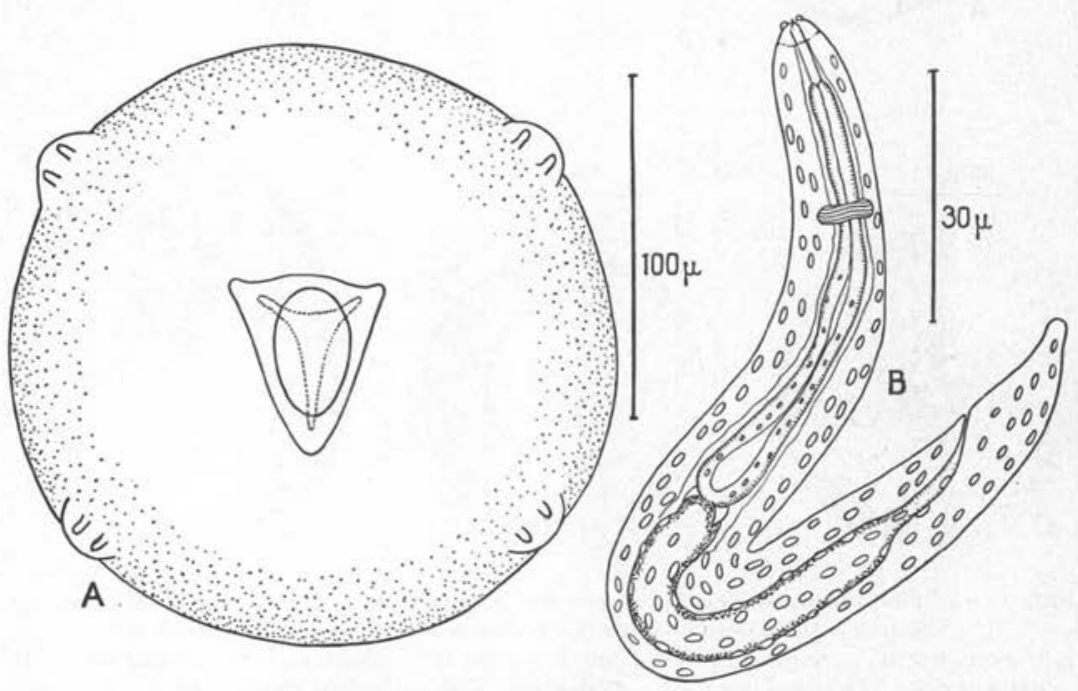

Fig. 3. - Seuratum tacapense (Seurat 1915)

A : Matériel type, parasite de Ctenodactylus gundi Pallas (Collection Seurat). Fcmelle. Extrémité céphalique, vue apicale. Seuratum mucronatum (Rud. 1809)

B : Matériel Biocca et Chabaud, parasite de Plecotus auritus (L.), Richelieu (France). Larve extraite de l'utérus.

graduelle. La distinction entre platymyaires (qui correspondent à peu près à méromyaires) et cœlomyaires (qui correspondent à peu près à polymyaires, ainsi que l'indiquent Chitwood et Chitwood, 1950, p. 9), est plus facile à établir.

Nous avons comparé brièvement la structure des genres Falcaustra, Seuratum, Cucullanus, Procamallanus et Camallanus, et constaté les faits suivants :

Falcaustra (fig. 4A), Cosmocercoidea typique, semble avoir la musculature la plus primitive, car c'est elle qui reste la plus pro- 

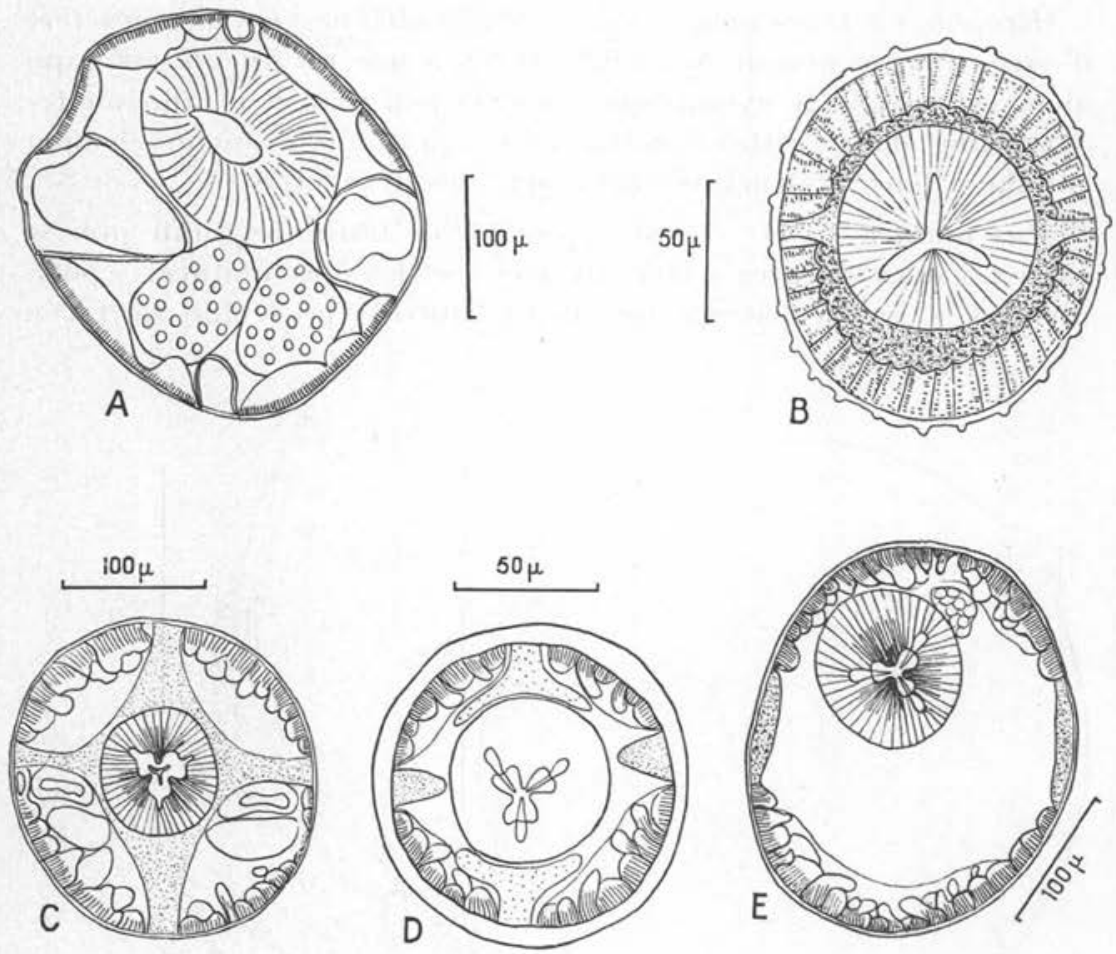

Fig. 4. - Schémas de différents types de musculature. Coupes au niveau de la région $\propto$ sophagienne ou de la portion antérieure de l'intestin.

A : Falcaustra golvani Chabaud et Brygoo 1957. Matériel type, parasite de Racophorus, Taanarive ; B : Seuratum mucronatum (Rud. 1809). Parasite de Plecotus auritus (L.), Pichelieu (France) ; C : Cucullanus hians (Dujardin 1845). Parasite de Conger conger (L.), Banyuls (France) ; D : Procamallanus levionchus (Weld 1862). Parasite de Synodontis schall (Bloch-Schneider), Congo belge; E : Cama!lanus elegans (Zeder 1800). Parasite de Perca fluviatilis L., Richelieu (France).

che de celle du Rhabditis (cf. illustration de Chitwood et Chitwood, 1950).

Seuratum (fig. 4 B) n'a plus les cellules musculaires nettement rassemblées en 2 ou 3 gros faisceaux dans chacun des quatre champs submédians, comme chez Falcaustra, mais présente une musculature homogène, formée de cellules qui sont toutes de taille égale. Il se rapproche d'Omeia (cf. Chabaud et Brygoo, 1957, fig. $4 \mathrm{D})$.

Cucullanus (fig. 4 C) s'en éloigne nettement, car les cellules musculaires sont inégales. Certaines sont petites, mais d'autres font 
saillie dans la cavité générale. Il y a donc une ébauche nette vers le type cœlomyaire.

Procamallanus (fig. 4 D) et Camallanus (fig. 4 E) ont une musculature inégale qui les rapproche de Cucullanus et des cœlomyaires.

Il semble donc que le caractère de musculature cœlomyaire, qui ne se trouve acquis que chez les Ascaridina les plus évolués, soit au contraire réalisé presque d'emblée chez les Spirurida. La musculature égale (platymyaire) du genre Seuratum, par opposition à celle déjà nettement inégale (cœlomyaire) du genre Cucullanus, représente done un bon argument pour penser que Seuratum est plus proche des Ascaridina que des Spirurida.

Anatomie génitale femelle: L'anatomie génitale femelle (voir Desportes, 1947), avec vagin relativement court et trajet complexe des tubes utérins, rappelle peut-être plus facilement celle des Ascaridina, mais aucun élément précis ne permet une orientation nette.

Anatomie génitale mâle: La queue du mâle sans ailes ou avec des ailes étroites, les papilles cloacales petites, nombreuses et non pédonculées, peuvent être rapprochées aussi bien des Cosmocercoidea que des Cucullans, mais le gros gubernaculum et les petits spicules sont typiques de certains Cosmocercides et non des Cucullanides.

Larves (fig. 3 B) : Nous avons pu voir les larves de Seuratum cadarachense Desportes, $S$. tacapense (Seurat) et $S$. mucronatum (Rud.), mais l'étude en est difficile sur ces spécimens fixés depuis très longtemps, et il serait important que la figure, que nous donnons à titre provisoire, soit vérifiée sur du matériel vivant.

Ce sont des larves trapues, à queue courte, s'opposant aux larves à long appendice caudal des Dracunculides et Camallanides. L'œsophage comprend une partie pharyngée étroite, un renflement antérieur peu prononcé, un isthme et un renflement postérieur. Il y a un crochet buccal. L'anatomie de la larve semble donc incompatible avec celle des larves de Spirurida et en parfait accord avec celle de certains Ascaridina, primitifs.

Cycle biologique: On ne connait rien sur la biologie des Seuratum ; il est cependant possible de remarquer que les hôtes intermédiaires connus chez les Camallina sont toujours des Copépodes. Il est bien peu probable que ces Crustacés puissent intervenir dans le cycle évolutif de parasites de Cheiroptères.

— Genre Skjabinura Gnédina 1933 (fig. 5).

Gnédina a classé son genre dans une famille distincte, mais son texte montre bien qu'elle a hésité, et que l'analogie avec les Seura- 
tidæ ne lui a pas échappé. Elle a éliminé le groupe des Ascaridina pour «l'absence de trois lèvres à l'extrémité céphalique et du bulbe œsophagien », mais l'on connaît maintenant de nombreux Ascaridina qui ont une bouche triangulaire sans lèvres et un œsophage sans bulbe, du même type que celui des Skrjabinura. Il est intéressant de constater que l'espèce de Gnédina a un gros gubernaculum et deux petits spicules, comme la plupart des Seuratidx. La

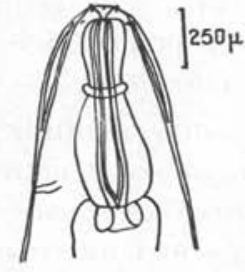

A
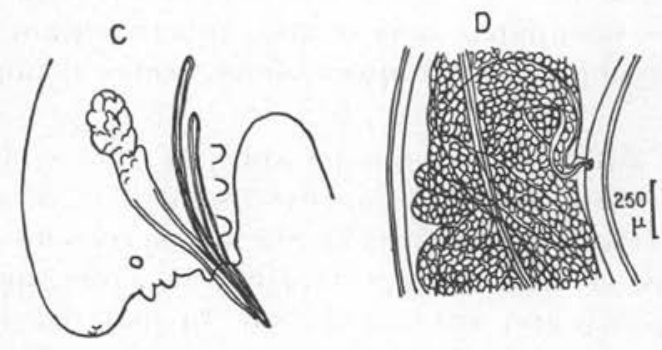

FIG. 5. - Skrjabiura spiralis Gnédina 1933. A : Extrémité antérieure de la femelle ; B : Extrémité postérieure de la femelle; C : Extrémité postérieure du mâle ; D : Région de la vulve. D'après Gnédina 1933.

mise en synonymie de Seuratinema Johnston et Mawson 1941a, faite par Thomas-Mawson (1960), renforce ce point de vue, puisque Seuratinema avait été considéré dès l'origine comme proche de Seuratum.

\section{- Genre Rictularina Johnston et Mawson $1941 b$ (fig. 6 B-C).}

Décrit d'après une femelle immature et une larve, le genre reste très peu connu, en dehors du fait qu'il existe des rangées d'épines cuticulaires. La forme de la tête et de l'œsophage n'a rien d'incompatible avec les genres précédents. 


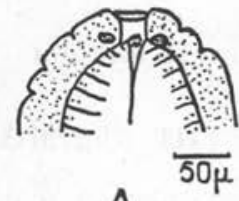

A

E

$\mathrm{D}$
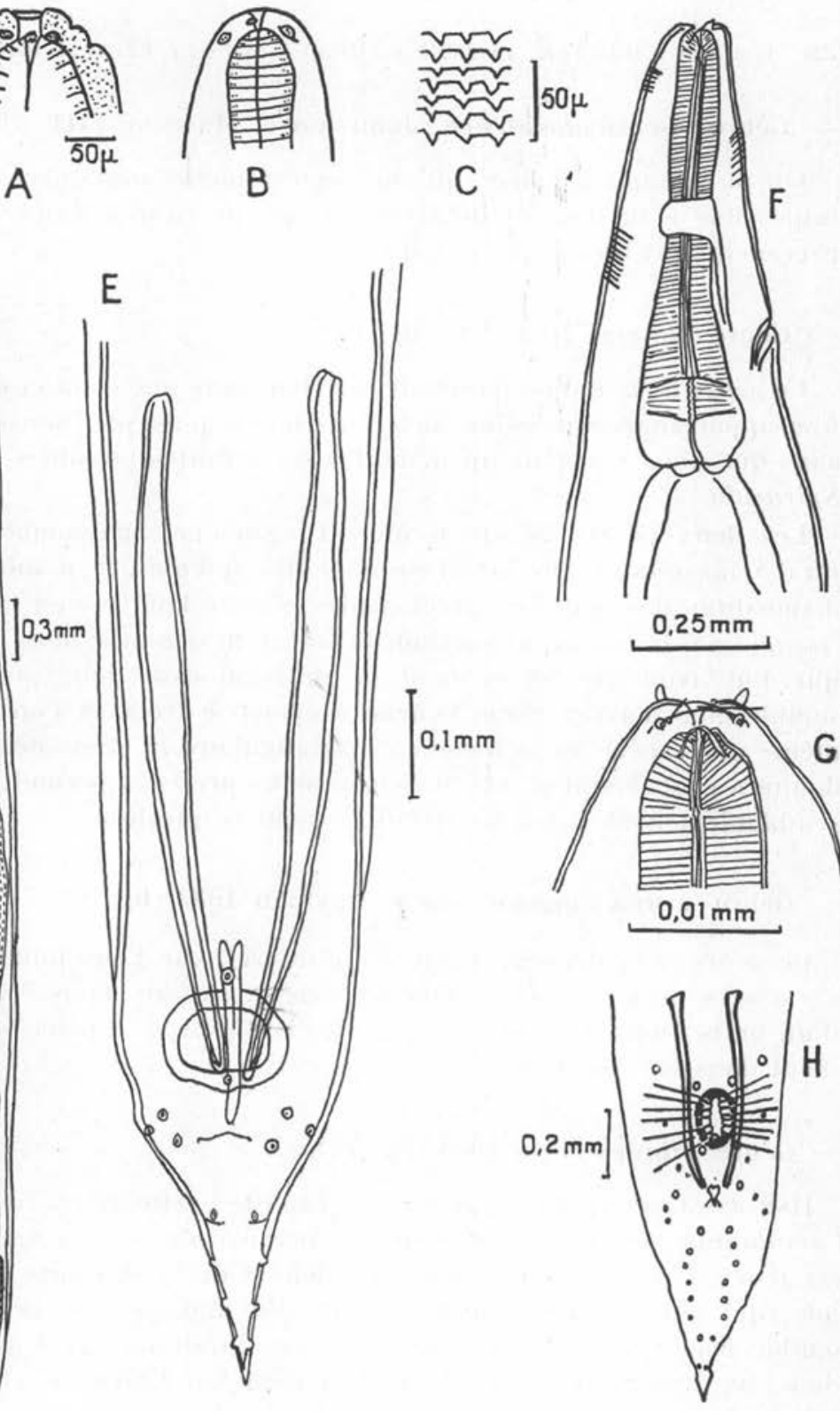

$10,3 \mathrm{~mm}$
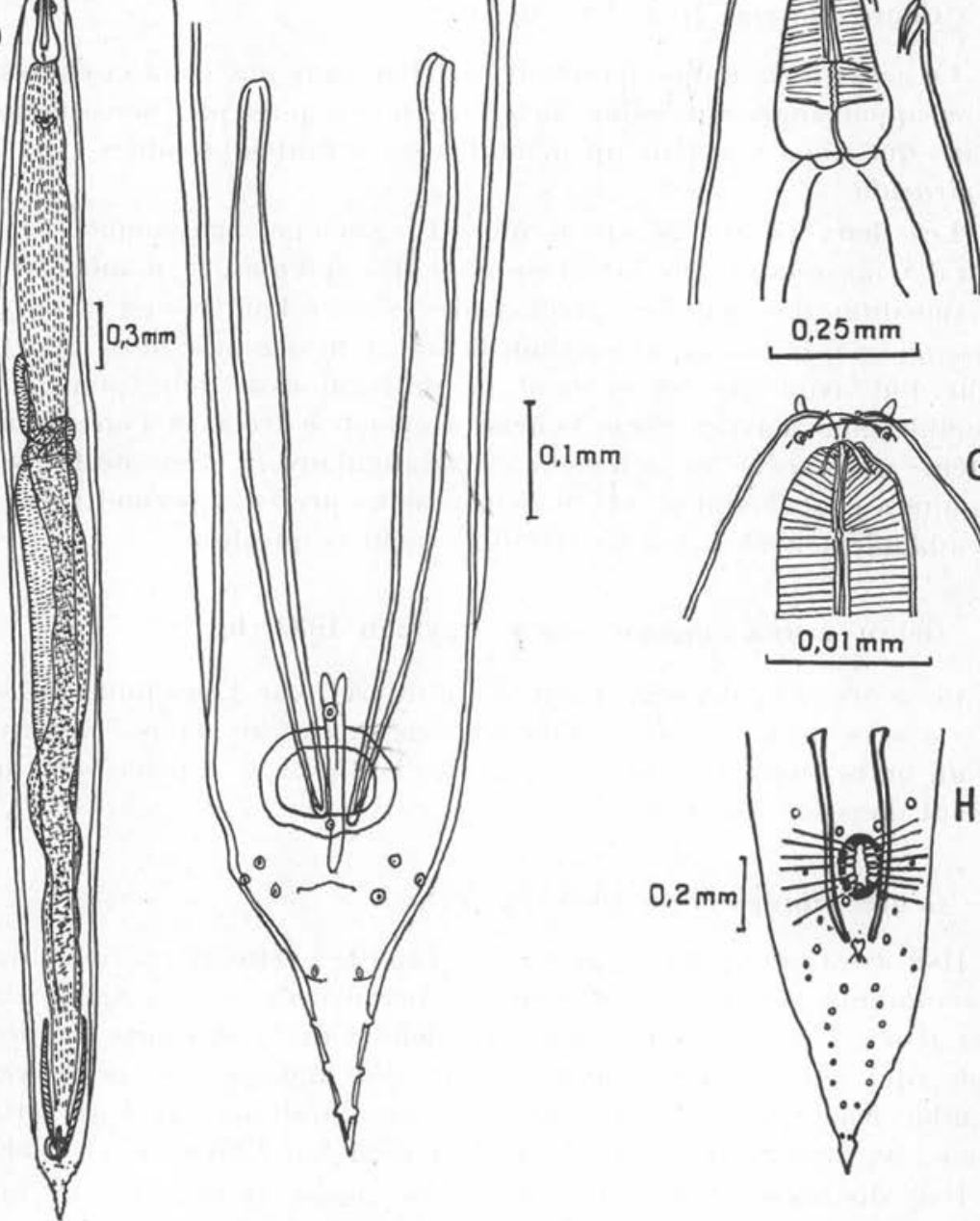

Fig. 6. - Denticulospirura dentata Johnston et Mawson 1941c

A : Extrémité antẻrieure. D'après Johnston et Mawson 1941c. Rictularina spinosa Johnston et Mawson $1941 b$

B : Extrémité antérieure ; C : Partie de la cuticule du milieu du corps.

D'après Johnston et Mawson $1941 b$.

Schneidernema retusa Rudolph, 1819

D : Mâle entier ; E : Extrémité postérieure du mâle, vue ventrale. D'après Travassos 1926 .

Morgascaridia sellsi (Morgan 1927)

F : Extrémité antérieure; G : Tête, vue dorsale; H : Extrémité postérieure du mâle, vue ventrale. D'après Inglis 1958. 
- Genre Denticulospirura Johnston et Mawson 1941c (fig. 6 A).

On ne connaît ici aussi qu'une seule femelle immature, et il est impossible actuellement de classer le genre ; une affinité avec les précédents est cependant possible.

\section{— Genre Omeia Hsü 1933 (fig. 16).}

Ce genre a été étudié en détail par Hsü, sans que celui-ci envisage une appartenance possible aux Ascaridina, puis par nous-mêmes, sans que nous pensions un instant à ses affinités possibles avec les Spirurida.

Les deux points de vue semblent également défendables, bien qu'il nous semble que la forme ailée des spicules, le nombre et la disposition des papilles précloacales s'accordent mieux avec les Ascaridina. En outre, et surtout, la forme hexagonale de la bouche, qui doit avoir été un élément de décision essentielle pour Hsü, semble bien dériver d'une bouche à symétrie trois, et l'on connaît même une espèce où la bouche est triangulaire. L'étude de la larve donnera probablement des renseignements précis et permettra vraisemblablement de résoudre définitivement la question.

\section{- Genre Cottocomephoronema Layman 1933 (fig. 12).}

Ce genre a également beaucoup d'intérêt, car l'ensemble de ses caractères permet de le classer près de Seuratum, mais l'existence d'un bulbe individualisé à l'arrière de l'œesophage le place certainement dans les Ascaridina.

\section{— Genre Pingus Hsü 1933 (fig. 11).}

Hsü avait créé pour ce genre une famille particulière, reliée aux Cucullanidæ, car il y a une ventouse précloacale, et aux Spiruridæ, car il n'y a pas de lèvres, mais, en dehors de la ventouse précloacale (qui n'a pas une grande valeur systématique), le genre ressemble beaucoup au précédent, et nous n'hésitons pas à le rattacher aux Seuratinæ, comme l'avaient déjà fait Chitwood et Wehr.

Hsü distingue dans l'œsophage une partie musculaire et une partie glandulaire, comme chez les Spirurides, mais, en réalité, cet œsophage court et cylindrique, pourvu d'une partie pharyngée bien différenciée, rappelle beaucoup plus l'œsophage d'un Kathlanide que l'œsophage d'un Spiruride. 
- Genre Haplonema Ward et Magath 1916 (fig. $13 \mathrm{~A}-\mathrm{B}$ ).

Ce genre a été confondu avec le précédent par Chitwood et Wehr, mais cette synonymie ne peut pas être conservée, car l'extrémité postérieure du mâle, au moins, est tout à fait différente. Les auteurs ont hésité entre Spiruroidea et Ascaroidea. Il semble bien que le genre puisse être rapproché du groupe des Seuratinæ. [Voir la description de Moulton (1931), et surtout la vue apicale de la tête (Chitwood et Chitwood, 1950, fig. $58 \mathrm{E}$ ), qui est presque identique à celle de Seuratum]. L'œsophage n'est pas très différent de Monhysterides, alors que, d'après Baylis et Daubney (1922), ce dernier genre appartient à un phylum tout à fait différent de celui qui nous intéresse ici.

\section{- Genre Ichtyobronema Gnédina et Savina 1930 (fig. 13 C-D).}

D'après Skrjabin (1949), le genre groupe trois espèces mal connues, mais Sudarikov et Ryjikov (1952) pensent qu'il n'y a qu'une espèce, d'ailleurs différente de celle de Linstow, qu'ils laissent comme "Spiroptère ». Ils rapprochent les genres Cottocomephoronema, Haplonema et Ichtyobronema dans la famille des Haplonematidæ (Thelazioidea).

\section{- Genre Paraseuratum Johnston et Mawson 1940.}

Il n'est connu que par des spécimens en mauvais état. Le genre a été rapproché de Seuratum par les auteurs, mais il y a lieu d'attendre des informations complémentaires pour pouvoir le classer, définitivement.

\section{— Genre Skrjabinelazia Sypliaxova 1930 (fig. 7).}

Skrjabinelazia est connu par deux espèces, l'une de Sypliaxova (1930), l'autre de Li (1934). Il a, jusqu'à maintenant, toujours été rangé dans les Thelaziidæ. Cependant, l'œsophage est court et simple; le mâle a un gros gubernaculum et deux petits spicules égaux comme dans les espèces précédentes. Enfin et surtout la tête a le type Ascaride et non Spiruride, car Li a vu une paire de papilles ventro-latérales incompatibles avec les Spirurida. La bouche triangulaire et la disposition concordent tout à fait avec Seuratum et nous proposons un rapprochement entre ces deux genres. Il faut cependant insister sur le caractère remarquablement synthétique de ce genre, car il ressemble non seulement aux Thelazia, mais encore aux Desmidocercides et à certaines Filaires, ce qui s'accorde parti- 
culièrement bien avec les vues d'Anderson sur la phylogénie des Filaires (1958).

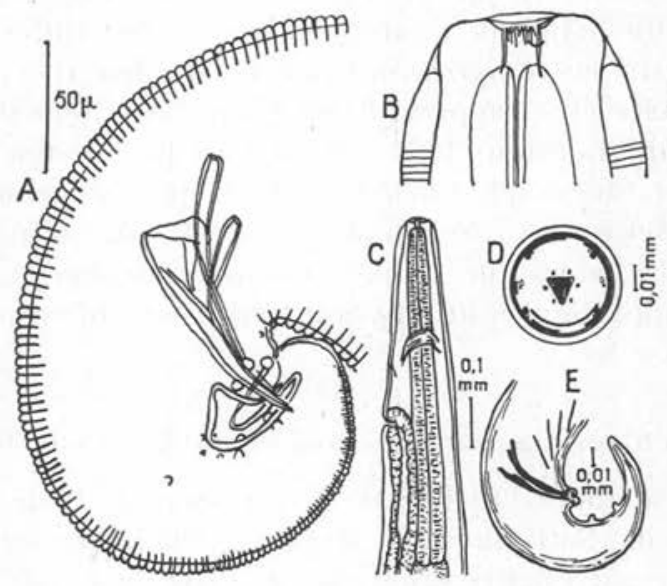

Fig. 7. - Skrjabinelazia taurica Sypliaxova 1930. A : Extrémité postérieure du mâle; B : Extrémité antérieure, bouclie avec coronule. D'après Sypliaxova 1930. - Skrejabinelazia hoffmani Li 1934. C : Extrémité antérieure, vue latérale de la femelle; D : Vue apicale de la femelle ; E : Extrémité postérieure mâle, vue latérale. D'après Li 1934.

\section{Conclusion}

En conclusion, par l'analyse détaillée du genre Seuratum, on constate que tous les caractères plaident de façon souvent très démonstrative, en faveur d'un rapprochement avec les Ascaridina. Le genre, classé correctement au début par Seurat et par Hall, s'est trouvé déplacé à cause d'un renseignement erroné sur la constitution céphalique, et la situation parmi les Spirurida semble avoir été conservée simplement parce que l'habitude en était prise. De nombreux genres ont été rattachés de façon plus ou moins explicite au genre Seuratum, et l'on constate en effet que, sous un aspect varié, ces genres ont des caractères communs, et ont toujours des éléments de type Ascaridina. Il existe même certains genres, tels que Skrjabinelazia, qui, bien qu'ayant toujours été considérés comme des Spirurida Thelaziidæ, ont cependant une paire de papilles céphaliques ventro-latérales typiques des Ascaridina et présentent également, par la structure céphalique, l'œsophage et l'anatomie génitale, des éléments communs avec Seuratum. 
La classification de Chitwood et Chitwood (1950), adoptée sans modifications très importantes par Skrjabin et coll. (1954), place à la base des Camallanoidea la famille des Camallanidæ.

Les Camallanides semblent effectivement des Nématodes archaïques, mais, par la symétrie bilatérale très évoluée de la tête et par l'œsophage musculo-glandulaire, ce sont des Spirurida. Ils sont, par rapport aux Spirurida, ce que sont les Kalicéphales par rapport aux Strongylina, c'est-à-dire un rameau riche en espèces et fortement spécialisé ; ils n'ont pas les éléments synthétiques que l'on peut espérer trouver à la base d'un phylum, et il nous semble préférable de mettre, à la base du sous-ordre, la famille des Cucullanidæ, plutôt que celle des Camallanidæ.

Dans notre conception, les Cucullanidæ sont des formes de passage entre Ascarides primitifs (Cosmocercoidea) et Spirurides. Les Seuratidx (probablement parce qu'ils sont parasites de Mammifères) ont toujours été considérés comme une branche évoluée des Cucullanidx, alors que nous pensons, au contraire, qu'ils sont plus proches des Ascarides et qu'ils ne peuvent pas être classés parmi les Spirurides. Les Cucullanidæ peuvent être considérés comme des Spirurides, car leur bouche a une symétrie bilatérale bien marquée et leur musculature n'est plus strictement platymyaire. Mais le cycle évolutif et même la morphologie larvaire restent inconnus et la symétrie buccale bilatérale reste très superficielle et se trouve remplacée dès le niveau de la capsule buccale par la symétrie d'ordre 3 des baguettes œsophagiennes (ef. Campana et Chabaud, 1957). Ils semblent en réalité se placer à la limite extrême du cadre des Spirurides et méritent donc une place particulière à la base du sous-ordre.

La troisième famille comprise dans les Camallanoidea: les Anguillicolidæ vivent essentiellement dans la vessie natatoire des Anguilles et ont un grand intérêt, car leur morphologie semble intermédiaire entre celle des Cucullanidæ (parasites intestinaux) et celle des Dracunculoidea (parasites tissulaires).

Nous croyons donc que le groupe Seuratum appartient aux Ascaridina, mais qu'il conduit aux Spirurida par l'intermédiaire de certains groupes et en particulier des Cucullanidæ.

B) Genres classés habituellement parmi les Quimperides:

— Genre Quimperia Gendre 1926 (fig. 14).

La bouche a trois lèvres basses, et entre donc nettement dans le cadre des Ascaridina. L'œsophage, entièrement musculaire, a cepen- 
dant une partie antérieure étroite et une partie postérieure élargie. La queue du mâle est pourvue d'une ventouse.

\section{- Genre Paraquimperia Baylis 1934 (fig. 15).}

Les lèvres sont plus petites que dans le genre précédent. L'œsophage a la même forme générale, mais il existe cependant une portion pharyngée, importante pour le rapprochement avec les Cosmocercoidea. Le mâle n'a pas de ventouse précloacale.

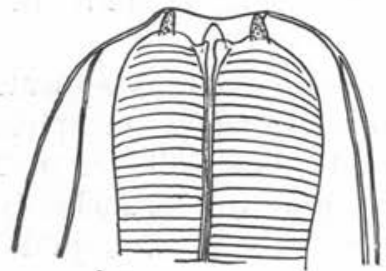

A

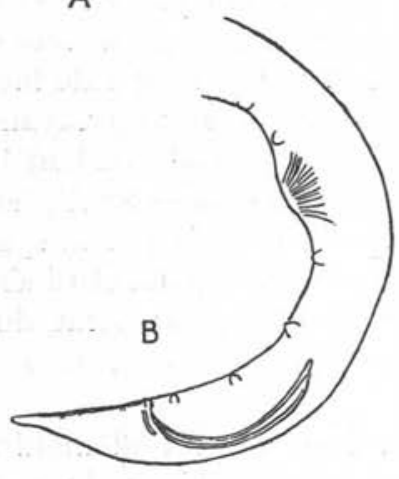

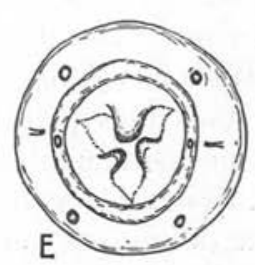
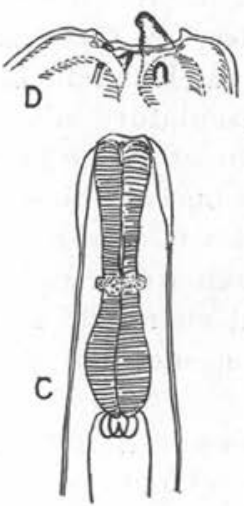

Fig. 8. - Gendria tilapiæ Baylis 1930. A : Tête de la femelle, vue ventrale ; B : Extrémité postérieure du mâle, vue latérale; C : Extrémité antérieure de la femelle, vue ventrale; D : Tête de la femelle, vue dorso-latérale gauche; $\mathrm{E}$ : Tête de la femelle, vue apicale. A, B, C, d'après Baylis 1930 ; D, E, d'après Chabaud 1956.

\section{- Genre Gendria Baylis 1930 (fig. 8).}

La bouche est presque circulaire et il n'y a pas de lèvres. Il existe trois fortes dents au fond de la cavité buccale. L'œsophage est claviforme. Le mâle possède une ventouse précloacale. 
- Genre Paragendria Baylis 1939 (fig. 9).

Gendre avait indiqué, dès 1926 , les affinités de l'espèce, mais c'est en 1933 seulement que Baylis a formé le genre Paragendria à partir de la description donnée par Stewart (1914). Il n'y a pas de lèvres, ni de dents à la base de la bouche, l'œsophage est claviforme. Le mâle possède une ventouse précloacale.

Fig. 9. - Paragendria macronis (Stewart 1914). A-B : Queue du mâle, vue ventrale ; $\mathrm{C}$ : Tête de profil, par plan à droite de la bouche ; D : Tête de profil, plan passant par la bouche; E : Ouverture ano-génitale et spicule gauche, vue latérale gauche ; F : Extrémité antérieure, vue médiane; G : Coupe transversale, en arrière du milieu du corps. D'après Stewart 1914 .

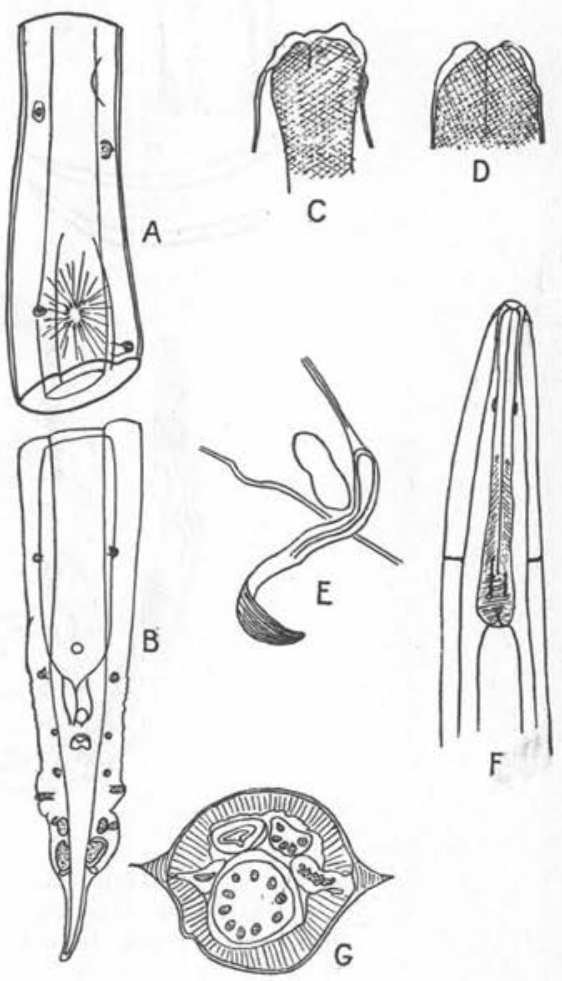

- Genre Buckleynema Ali et Singh 1954 (fig. 10).

Le genre est proche de Paragendria et de Gendria. Il en diffère surtout par la dentition de la capsule buccale et par l'existence de quatre papilles céphaliques médio-médianes qui n'existent pas chez Gendria. 

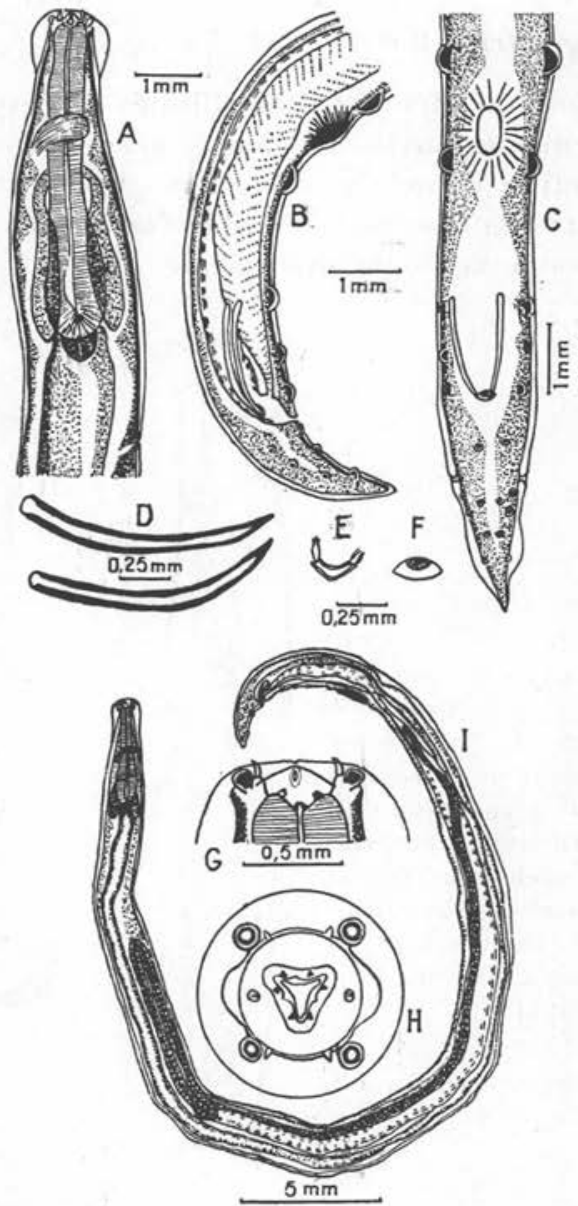

FIG. 10. - Bucklegnema buckleyi Ali et Singh 1954. A : Extrémité antérieure, vue latérale ; B : Extrémité postérieure du inâle, vue latérale; C : Extrémité postérieure du mâle, vue ventrale ; D : Spicules, vue latérale ; E : Gubernaculum et pointes des spicules; F: Cloaque du mâle avec papille préanale; G: Tête, vue latérale; $\mathbf{H}$ : Tête, vue apicale; I : Mâle, vue latérale. D'après Ali et Singh 1954.

\section{Conclusion}

Les genres Seuratum et Quimperia ne se ressemblent pas, et, bien que le rapprochement entre Quimperiidæ et Cucullanidæ ait été fait à de nombreuses reprises, le rapprochement entre Quimperiidæ et 
Seuratidæ n'a pas, à notre connaissance, été proposé. Pourtant, il semble difficile de donner à la famille des Seuratidæ une meilleure définition que celle qui est donnée par Baylis (1939) pour la famille des Quimperiidæ. (On peut y corriger seulement que la capsule buccale est présente dans le genre Omeia, que certains genres sont vivipares plutôt qu'ovipares, et que le bulbe œsophagien reste légèrement individualisé dans le genre Cottocomephoronema). Il est donc tout à fait impossible de conserver les deux familles dans leur sens actuel, car elles ont pratiquement la même définition, et on ne voit aucun élément susceptible de permettre une bonne division en deux parties distinctes.

\section{G) Genres classés habituellement parmi les Hétérakides :}

En collaboration avec W. Inglis (1960), nous avons indiqué tout récemment les raisons qui nous incitent à proposer le rattachement des genres Schneidernema (fig. $6 \mathrm{D}, \mathrm{E}$ ) et Morgascaridia (fig. $6 \mathrm{~F}, \mathrm{H}$ ) aux groupes précédents.

\section{D) Place des SEURATOIDEA}

Par la morphologie de l'adulte et par la morphologie larvaire, chaque fois que celle-ci est connue, le groupe des Seuratoidea doit être rattaché aux Ascaridina, dans le cadre ou tout à côtté des Cosmocercoidea. Cette superfamille semble donc à l'origine non seulement de tous les Ascaridina (cf. Chabaud, 1957), mais encore des Spirurida, par l'intermédiaire des Seuratidæ-Quimperiidæ.

En effet, les Cucullanidæ étroitement liés à eux peuvent cependant être rangés dans les Camallanina, et c'est à partir de formes proches que l'on peut concevoir un rameau fortement spécialisé à la vie intestinale (Camallanidæ) et un phylum s'adaptant à la vie tissulaire (Anguillicolidæ, puis Dracunculidx). En outre, il est extrêmement frappant de voir que, parmi les Seuratidæ-Quimperii$d x$, il existe des genres tels que Ichtyobronema qui ont été rapprochés de Rhabdochona (Thelaziidæ, Spiruroidea), et Omeia ou Skrjabinelazia qui, d'après la morphologie de l'adulte, ont été classés sans hésitation comme des Thelaziidæ (1). On sait que c'est précisément cette famille des Thelaziidæ qui, de l'avis de Chitwood et Wehr, est à l'origine des Spiruroidea et des Filarioidea. Nous cherchons, dans le schéma ci-joint, à rendre compte des affinités que nous concevons pour ce groupe qui formerait ainsi, dans le cadre des Ascaradina, la base des Spirurida primitifs (fig. 17).

(1) Si l'on admet qu'Omeia se rapproche des ancêtres des Thelaziidr, les six lèvres des Thelaziides actuels ne correspondent pas, comme on le croyait jusqu'à maintenant, aux six lèvres des Rhabditis. 


\section{Modifications systématiques proposées}

L'ensemble ainsi formé évoque tout de suite un groupe archaïque, car il y a presque un genre séparé pour chaque espèce, et celles-ci sont réparties dans le monde entier chez les animaux les plus variés (essentiellement Poissons, mais aussi Amphibiens, Reptiles, Oiseaux, Mammifères). Cette notion vient à l'appui des conclusions formulées plus haut et nous conduit à rapprocher ce groupe des Ascaridina les plus primitifs, c'est-à-dire des Cosmocercoidea. C'est à cette conclusion que nous avait conduit déjà l'étude du genre Omeia, et les affinités de ces familles avec les Kathlanides (c'est-àdire, pour nous, les Cosmocercoidea) ont été soulignées depuis très longtemps, en particulier par Baylis.

Nous aurions donc voulu rassembler tous ces genres en une seule famille, et la placer dans la superfamille des Cosmocercoidea, mais nous nous heurtons à une difficulté qui semble insurmontable, car les formes qui se trouveraient rassemblées dans cette superfamille (Cosmocerca, Kathlania, Quimperia, Seuratia) sont tellement variées qu'il devient pratiquement impossible d'en donner une définition. Malgré le nombre très limité des espèces, nous croyons donc que la seule solution possible est de créer une superfamille particulière, Seuratoidea.

Les 18 genres de cette superfamille forment difficilement des groupes homogènes et l'on ne peut pas conserver la division en deux familles, car aucune limite nette ne permet de les scinder en deux parties. Nous préférons done les rassembler tous dans la seule famille des Seuratidæ.

Les genres Seuratum (fig. 1-3) d'une part et Skrjabinura (fig. 5) d'autre part sont très différents, mais ils ont cependant en commun un œsophage simple, une queue courte et des œufs contenant des larves au moment de la ponte. Nous préférons donc les rassembler en une seule sous-famille, car les coupures supra-génériques sont déjà trop nombreuses pour un groupe qui compte aussi peu d'espèces.

Schneidernema (fig. $6 \mathrm{D}, \mathrm{E}$ ) et Morgascaridia (fig. $6 \mathrm{~F}, \mathrm{H}$ ) peuvent être groupés sans difficultés. Ils se différencient des précédents par l'existence d'une ventouse précloacale et par le fait que les œufs ne sont pas embryonnés.

Le genre Skrjabinelazia (fig. 7) se rapproche des précédents, mais la vulve est très antérieure, la queue est longue, l'œsophage plus 
grêle et il est parasite de Reptile. Il semble donc qu'il faille le séparer dans une sous-famille particulière.

Le genre Omeia (fig. 16) a tellement de caractères particuliers qu'il semble nécessaire de le traiter séparément.

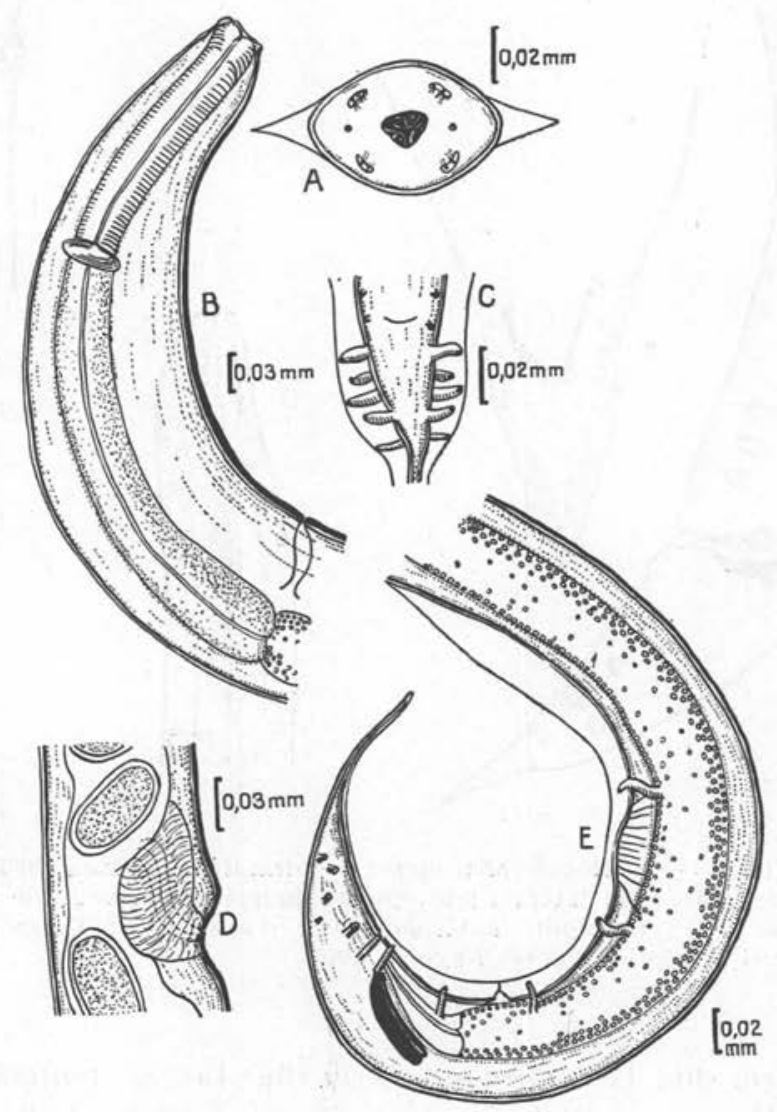

FIG. 11. - Pingus sinensis Hsü 1933. A : Tête, vue apicale; B: Extrémité antérieure, vue latérale; C: Mâle, extrémité postérieure, vue ventrale; D : Femelle, vulve et œufs; E : Queue du mâle, vue latérale. D'après Hsü 1933.

Cottocomephoronema (fig. 12), Pingus (fig. 11), et Haplonema (fig. $13 \mathrm{~A}, \mathrm{~B}$ ) ont en commun un œsophage cylindrique, mais toujours nettement divisé en deux portions. Nous les rassemblons donc tous les trois. 

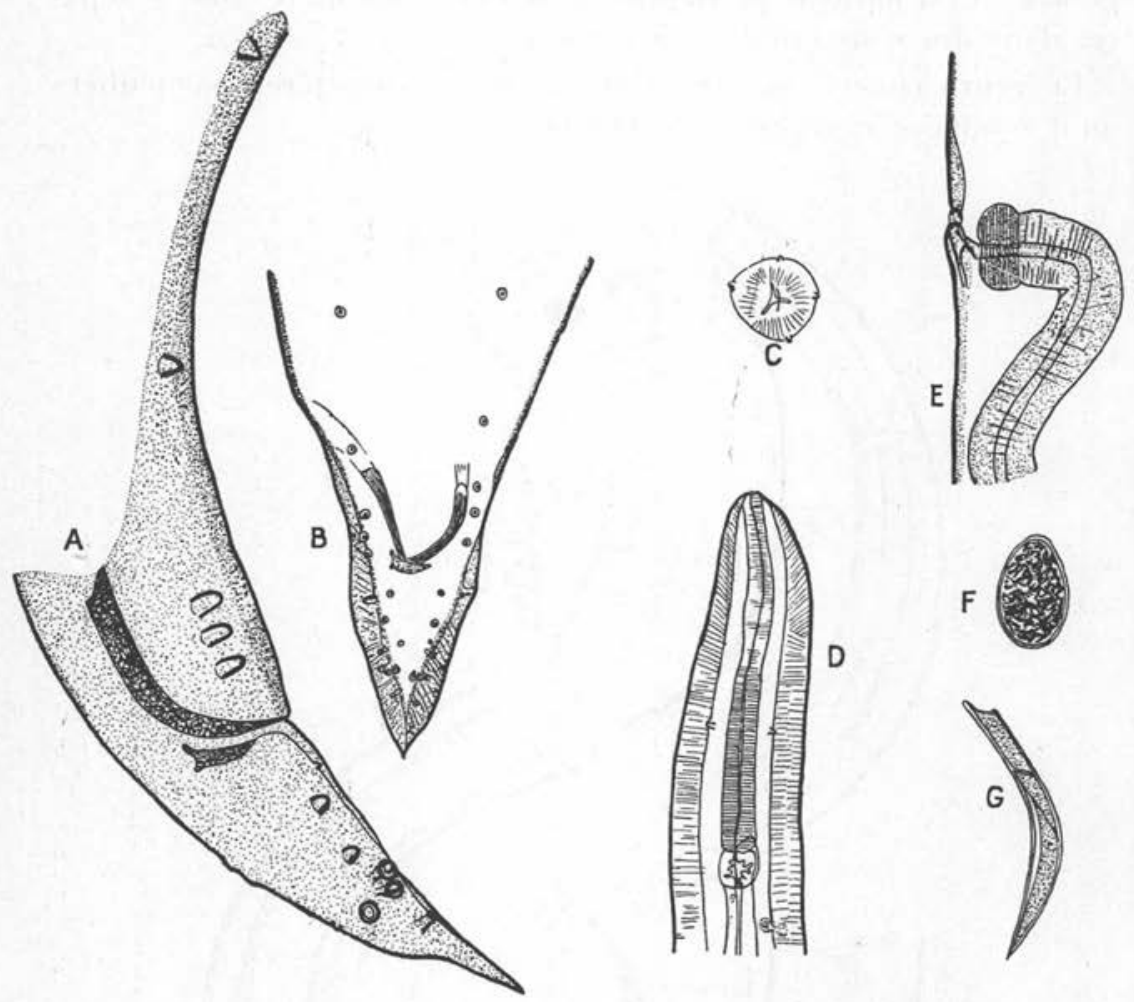

Fig. 12. - Cottocomephoronema problematica Layman 1933

A : Queue du mâle, vue latérale ; B : Queue du mâle, vue ventrale ; C : Tête, vue apicale; D : Extrémité antérieure, vue dorsale ; E : Vulve et vagin ; F : GEuf ; G : Spicule. D'après Layman 1933.

Quimperia (fig. 14), Paraquimperia (fig. 15) et Ichtyobronema (fig. $13 \mathrm{C}, \mathrm{D}$ ) ont, au contraire, la moitié postérieure de l'œsophage fortement enflée. Ce sont les seuls qui aient des lèvres légèrement indiquées.

Enfin, Gendria (fig. 8), Paragendria (fig. 9) et Buckleynema (fig. 10) ont tous trois un œsophage cylindrique non divisé.

Nous croyons donc pouvoir retenir sept sous-familles, basées essentiellement sur la forme de l'œsophage, et nous proposons les définitions suivantes : 


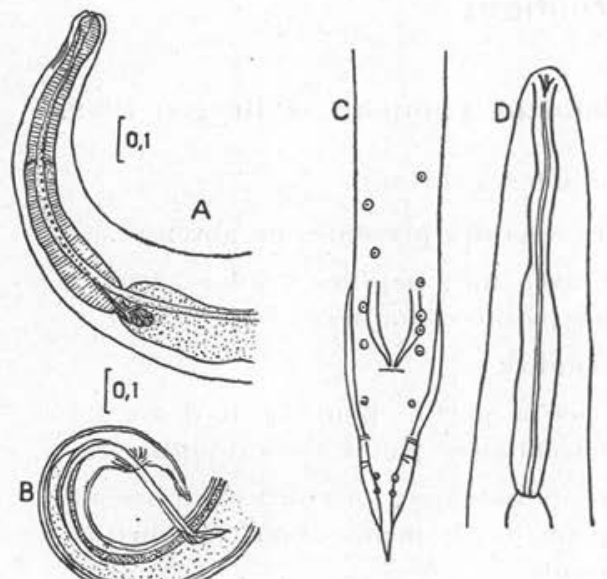

Fig. 13. - Haplonema immutatum Ward et Magath 1916. A : Extrémité antérieure de la fernelle; B : Queue du mâle. D'après Ward et Magath 1916. - Ichtyobronema conoura Linstow 1885. C : Queue du mâle; D : Extrémité antérieure. D'après Gnédina et Ssavina 1930 in Skrjabin et coll. 1949.

FIG. 14. - Quimperia lanceolata Gendre 1926. A : Extrémité antérieure, vue médiane; B: Région œsophagienne; C : Tête, vue dorsale; D : Tête, vue ventrale;
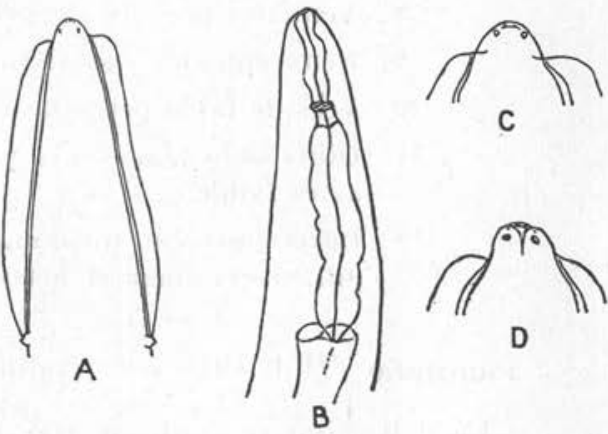
E : Queue du mâle, vue latérale. D'après Gendre 1926.

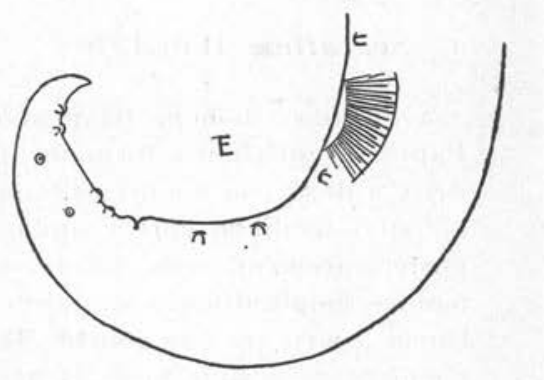




\section{Définitions}

SEURATOIDEA (Hall 1916 s.-f.) Chabaud, Campana et Brygoo 1959.

Ascaridina ayant la plupart des caractères suivants :

1. Papilles céphaliques ventro-latérales présentes ou absentes.

2. Cycle céphalique externe avec huit papilles soudées deux à deux, plus rarement quatre papilles simples.

3. Lèvres absentes ou très réduites.

4. Capsule buccale faible et petite, parfois pourvue de dents, ou grande, triangulaire, et non entourée par le tissu œsophagien.

5. Esophage court, simple et cylindrique, ou court et divisé en deux parties ayant ou n'ayant pas le même diamètre. Portion pharyngée présente ou absente.

6. Musculature platymyaire.

7. Cæcum digestif absent, plus rarement présent.

8. Ventouse précloacale présente ou absente.

9. Deux spicules égaux. Gubernaculum souvent très grand.

10. Vers de taille petite ou moyenne, parasite de Vertébrés.

11. CEufs embryonnés à coque mince, ou non embryonnés à coque assez faible.

12. Développement inconnu. Peut-être premiers stades dans le milieu extérieur et hétéroxénie.

Seuratidæ (Hall 1916 s.-f.) Raillet 1916.

La définition se confond avec celle de la superfamille.

\section{a) Seuratinæ Hall 1916 (= Skrjabinuridæ Gnédina 1933).}

Seuratida; bouche triangulaire sans lèvres. Pas de capsule buccale. Papilles céphaliques formant un cycle externe de huit papilles groupées deux à deux; la médio-médiane au même niveau et de même taille que la latéro-médiane correspondante. Esophage simple, très court, enflé postérieurement, sans différenciation pharyngée. Cuticule avec ou sans bandes longitudinales spinulées. Ailes cervicales présentes ou absentes. Queue courte ou très courte. Mâle sans ventouse précloacale, avec gubernaculum de grande taille et deux spicules petits et égaux. Femelle avec vulve équatoriale ou pré-équatoriale. OEufs à coque mince, complètement embryonnés. Parasites de Mammifères et d'Oiseaux. 
Genres : Seuratum Hall 1916, Skrjabinura Gnédina 1933 (= Seuratinema Johnston et Mawson 1941a), (?) Denticulospirura Johnston et Mawson 1941c, et (?) Rictularina Johnston et Mawson $1941 b$. (Fig. 1-6c).

\section{b) Schneidernematina Freitas 1956 .}

Seuratidæ: bouche triradiée avec lèvres peu développées. Capsule buccale faible, pouvant cependant être armée d'une denticulation. Quatre papilles céphaliques doubles. Esophage simple, très court, enflé postérieurement, avec différenciation pharyngée. Ailes latérales présentes ou absentes. Queue courte. Mâle avec ventouse précloacale, gubernaculum assez petit, et deux spicules égaux. Femelle avec vulve pré-équatoriale. CEufs non embryonnés. Parasites de Mammifères.

Genres : Schneidernema Travassos 1927 et Morgascaridia Inglis 1958. (Fig. 6 D-H).

\section{c) Skrjabinelaziinz Chabaud, Campana et Brygoo 1959.}

Seuratidæ : bouche triangulaire sans lèvres. Capsule buccale très faible avec parfois une couronne de petites dents. Papilles céphaliques formant un cycle externe de dix papilles : quatre médio-médianes, quatre latéromédianes et deux ventro-latérales, toutes sur le même niveau, ainsi que les amphides. Esophage simple, court et régulièrement cylindrique, sans différenciation pharyngée. Cuticule lisse, sans ailes, parfois enflée dans la région cervicale. Queue longue. Mâle sans ventouse précloacale, avec gubernaculum relativement grand et deux spicules petits et peu inégaux. Femelle avec vulve très antérieure, au niveau de l'œsophage. Eufs à coque moyennement épaisse, complètement embryonnés. Parasites de Reptiles.

Genre unique : Skrjabinelazia Sypliaxova 1930. (Fig. 7).

\section{d) Gendriinz Chabaud, Campana et Brygoo 1959.}

Seuratidæ: bouche triangulaire ou circulaire sans lèvres. Capsule buccale très faible, la base pouvant cependant être armée de dents. Papilles céphaliques variables suivant les genres. EEsophage simple, très court, enflé postérieurement, sans différenciation pharyngée. Cuticule cervicale formant une forte dilatation vésiculeuse. Ailes latérales présentes ou absentes. Mâle avec ventouse précloacale. Spicules égaux. 
Gubernaculum petit ou absent. Femelle avec vulve en arrière du milieu du corps. Eufs. non embryonnés. Parasites de Poissons.

Genres : Gendria Baylis 1930, Paragendria Baylis 1939 et Buckleynema Ali et Singh 1954. (Fig. 8-10).

e) Pinginz (Hsü 1933 fam.)

(= Haplonematidæ Sudarikov et Ryjikov 1952).

Seuratidæ: bouche triangulaire sans lèvres. Pas de capsule buccale. Papilles céphaliques formant un cercle externe de huit papilles groupées deux à deux, la médio-médiane au même niveau que la latéro-médiane correspondante, ou (?) quatre papilles. Esophage cylindrique court, la moitié postérieure ayant une structure différente de la moitié antérieure. Portion pharyngée parfois différenciée. Parfois, bulbe creux présent à l'arrière de l'œsophage. Ailes latérales fortes, surtout dans la région cervicale. Mâle avec ou sans ventouse précloacale. Deux spicules égaux. Gubernaculum assez petit ou absent. Ailes caudales parfois légèrement développées. Femelle avec vulve équatoriale ou post-équatoriale. Eufs non embryonnés. Parasites de Poissons.

Genres : Pingus Hsü 1933, Cottocomephoronema Layman 1933 et Haplonema Ward et Magath 1916. (Fig. 11-13).

\section{f) Quimperiinæ Gendre 1928 .}

Seuratidæ: bouche entourée de trois lèvres basses, semi-globuleuses, parfois très petites. Capsule buccale très faible, la base pouvant porter de petites dents. Quatre papilles céphaliques. Esophage cylindrique, avec tiers antérieur nettement plus étroit que les deux tiers postérieurs. Portion pharyngée parfois bien différenciée. Ailes cervicales et latérales généralement bien développées. Mâle avec ou sans ventouse précloacale. Deux spicules égaux. Gubernaculum assez petit ou (?) absent. Ailes caudales parfois légèrement développées. Femelle avec vulve postéquatoriale. Eufs non embryonnés. Parasites de Poissons.

Genres: Quimperia Gendre 1926, Paraquimperia Baylis 1934, Ichtyobronema Gnédina et Ssavina 1930, et (?) Paraseuratum Johnston et Mawson 1948. (Fig. 14-15). 


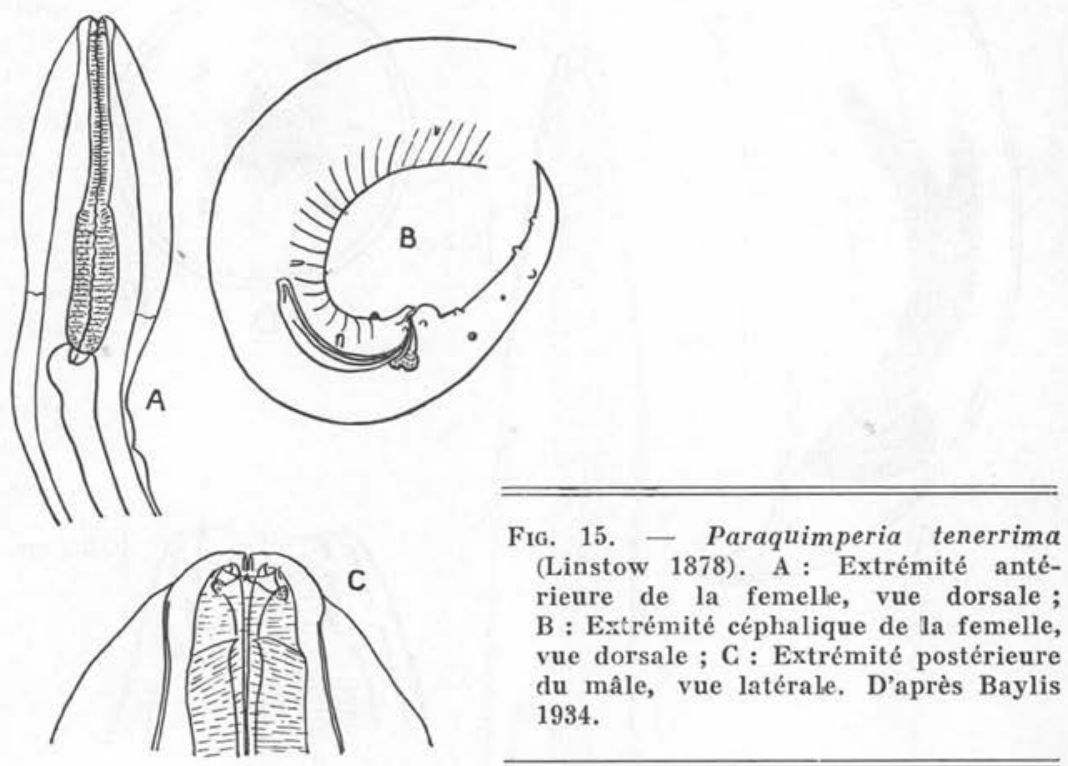

\section{g) Omeiina Sobolev 1949.}

Seuratidæ: bouche hexagonale ou triangulaire, finement denticulée. Capsule buccale prismatique triangulaire, assez grande et bien chitinisée, non entourée de tissu œsophagien. Huit papilles céphaliques submédianes groupées deux à deux. Papilles ventro-latérales absentes. Esophage cylindrique, simple. Intestin pourvu d'un cæcum creux. Ailes latérales présentes. Mâle sans ventouse précloacale. Deux spicules égaux et ailés. Gubernaculum présent, souvent grand. Femelle avec vulve à peu près équatoriale. CEufs non embryonnés. Parasites d'Amphibiens.

Genre unique : Omeia Hsü 1933 (= Harantinema Chabaud et Brygoo 1957) (1). (Fig. 16).

(1) Omeia amboceca (Chabaud et Brygoo 1957) se distingue facilement d'O. papillocauda Rankin 1937, parasite d'Urodèle d'Amérique du Nord, par ses dimensions beaucoup plus fortes. Nous n'avons pas pu consulter la deseription d'O. chiekasans Walton 1940, parasite d'un autre Urodèle également d'Amérique du Nord. $O$. ambocrea est très proche d'O. hoepplii Hsü 1933, parasite de Rana en Chine. Chez ambocæca la bouche est un peu allongée dorso-ventralement, le gubernaculum relativement plus gros, la première paire de papilles postcloacales plus antérieure.

Ann. ne Parasitologie, T. XXXV, $\mathrm{N}^{\circ} 3 .-1960$ 


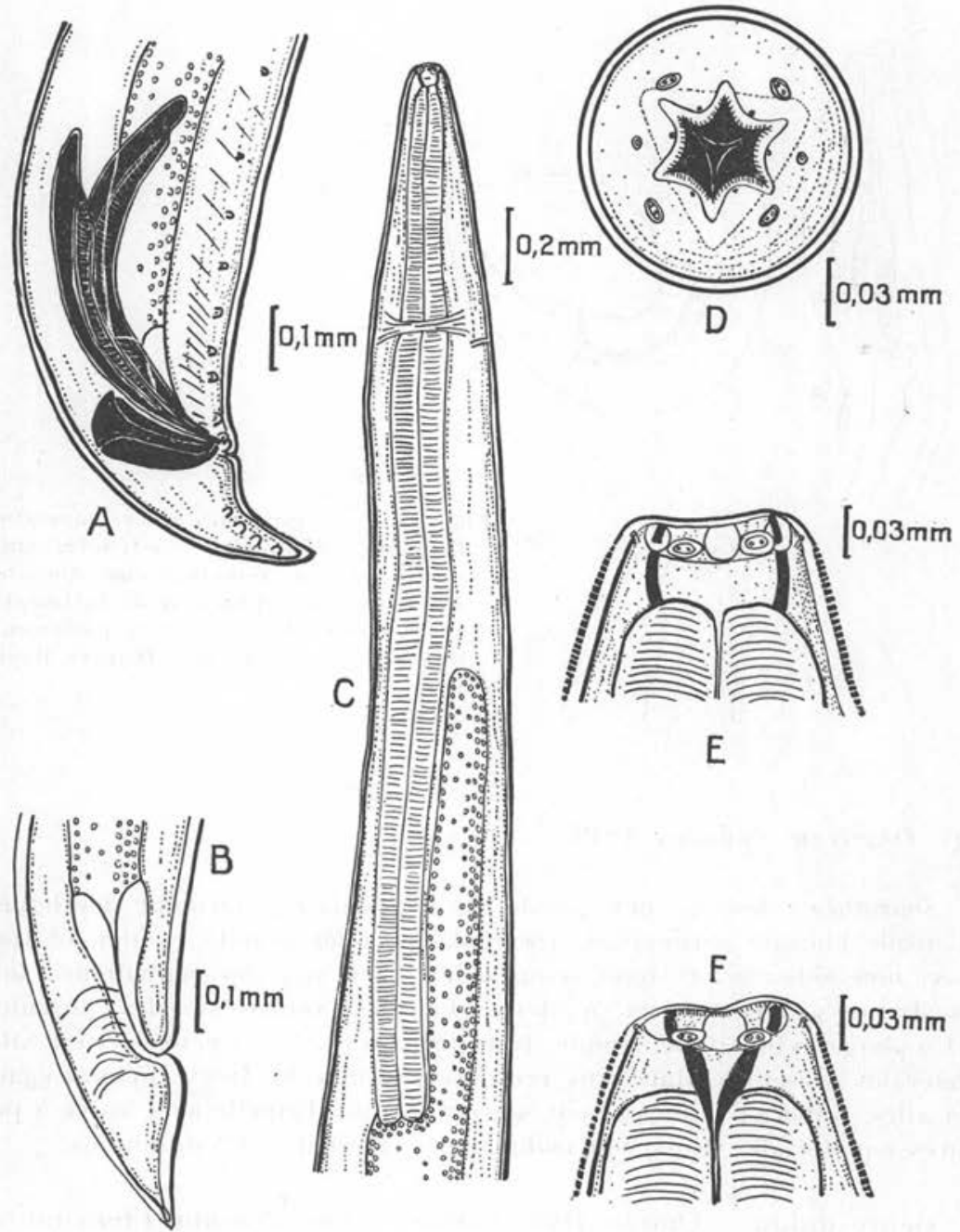

Fıg. 16. - Omeia hoepplii Hsü 1933

A : Mâle, extrémité postérieure, vue latérale ; B : Femelle, extrémité postérieure, vue latérale ; C : Extrémité antérieure, vue latérale ; D : Tête, vue apicale ; E : Tête, vue dorsale ; F : Tête, vue ventrale. D'après Hsü 1933.

\section{Résumé et Conclusions}

$1^{\circ}$ Le genre Seuratum et les genres voisins ont une morphologie qui les rattache aux Ascaridina et non aux Spirurida. La morphologie larvaire, en particulier, ne permet aucun doute. 
$2^{\circ}$ Les Cucullanidx, dont on ne connaît pas les larves, peuvent être à la rigueur considérés comme des Spirurida, car la tête présente une symétrie bilatérale, mais ils sont à la limite extrême entre Spirurida et Ascaridina.

$3^{\circ}$ Aucun élément ne permet de séparer la famille des Quimperiidæ de la famille des Seuratidæ.

$4^{\circ}$ Nous groupons les deux familles en une seule famille : les Seuratidæ, et nous proposons pour elle la nouvelle superfamille des Seuratoidea, qui entre dans le cadre des Ascaridina et a, pensons-

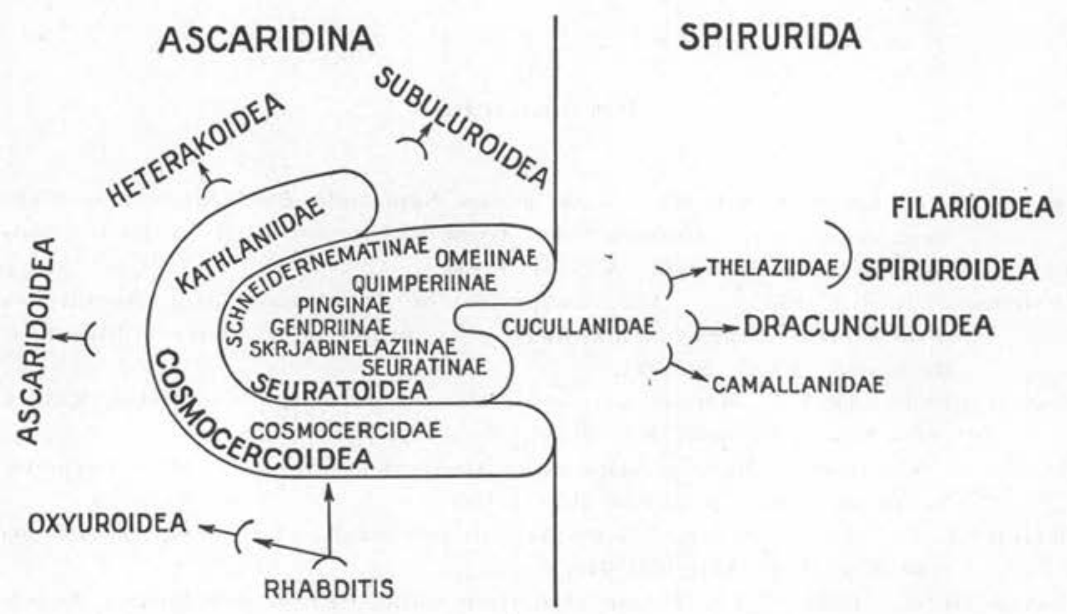

Fig. 17. - Schéma de taxonomie proposée pour la super-famille des Seuratoidea.

nous, des relations étroites avec les Cosmocercoidea (cette superfamille des Cosmocercoidea représentant précisément, dans notre conception, le groupe le plus généralisé des Ascaridina).

Les Seuratoidea semblent former un groupe de transition entre les Ascaridina et les Spirurida les plus primitifs, et être à l'origine d'une part des Camallanina (Camallanidæ adaptés à la vie intestinale, Dracunculoidea adaptés à la vie tissulaire), et d'autre part des Thelaziidæ. Il existe en effet des genres tels que Omeia qui ont été considérés comme des Thelaziidæ, alors que, pour nous (genre Harantinema), ce sont des Ascaridina.

$5^{\circ}$ Nous proposons de diviser la famille des Seuratidæ en sept sous-familles : 
a) Seuratinæ (=Skrjabinuridæ) ; genres : Seuratum, Skrjabinura (= Seuratinema), (?) Denticulospirura, (?) Rictularina.

b) Schneidernematinæ; genres : Schneidernema et Morgascaridia.

c) Skrjabinelaziinæ; genre : Skrjabinelazia.

d) Gendriinæ; genres : Gendria, Paragendria, Buckleynema.

e) Pinginæ; genres : Pingus, Cottocomephoronema, Haplonema.

f) Quimperiinæ ; genres : Quimperia, Paraquimperia, Ichtyobronema, (?) Paraseuratum.

g) Omeiinæ; genres : Omeia (= Harantinema).

\section{Bibliographie}

ALI (S. M.) et SINGH (S. N.), 1954. - On a new Nematode, Buckleynema buckleyi Gen. et sp. nov. (Quimperiidx) from a Siluroid Fish in HyderabadDeccan. J. of Helminth., XXVIII, 17-24.

Anderson (R. C.), 1957. - The life cycles of Dipetalonematid Nematodes (Filarioidea, Dipetalonematide) : the problem of their evolution. J. Helminth., XXXI, 203-224.

BAyLis (H. A.), 1923. - Report on a collection of parasitic Nematodes, Mainly from Egypt. Parasit., XV, 24-38.

Baylis (H. A.), 1930. - Mission Saharienne Augiéras-Draper, 1927-1928. Parasitic Nematodes. Bull. Museum, II, 117-130.

Baylis (H. A.), 1934. - A little-known Nematode Parasite of the Eel. Ann. Magazine Nat. Hist., XIII, 235-240.

Baylis (H. A.), 1939. - The Fauna of British India, Ceylon and Burma. Nematoda, vol. II, British Museum. London, 1939, xxvm + $274 \mathrm{pp}$.

Baylis (H. A.) et DaUbNey (R.), 1922. - Report on the parasitic nematodes in the collection of the zoological Survey of India. Mem. Indian Museum, VII, 263-347.

Baylis (H. A.) et DaubNey (R.), 1926. - A synopsis of the Families and genera of Nematoda. British Museum. London, 1926, xxxvi +276 pp.

Biocca (E.) et Chabaud (A. G.), 1951. - Redescription de Seuratum mucronatum (Rud. 1809) (Nematoda, Cucullanide). Ann. Parasit., XXVI, 86-92.

Campana-Rouget (Y.) et Chabaud (A. G.), 1956. - Helminthes des environs de Banyuls. III. Sur trois espèces de Cucullanus (Camallanoidea, Nematoda) parasites des Poissons. Vie et Milieu, VII, 267-356.

Сhabaud (A. G.), 1956. - Structure céphalique de Gendria tilapia Baylis 1930. Ann. Parasit., XXXI, 310-311.

Chabaud (A. G.), 1957. - Sur la systématique des Nématodes du sous-ordre des Ascaridina parasites des Vertébrés. Bull. Soc. Zool. France, LXXXII, 243-253.

Сhabaud (A. G.) et Brygoo (E. R.), 1957. - Deux nématodes parasites de Grenouilles à Madagascar : Falcaustra golvani n. sp. et Harentinema ambocara n. gen., n. sp. Ann. Parasit., XXII, 385-397. 
Cihabaud (A. G.), Campana-Rouget (Y.) et Brygoo (E. R.), 1959. - Les Nématodes Seuratoidea nov. sup. fam. et l'origine des Spirurida. C.R. Acad. Sc., CCXI.VIII, 1449-1451.

Chitwoon (B. G.) et Chiтwood (M. B.), 1950. - An Introduction to Nematology. Section I, Anatomy. Baltimore, 1950, vilI +213 pp.

Chitwood (B. G.) et WeHr (E. E.), 1934. - The value of cephalic structures as characters in nematode classification, with special reference to the superfamily Spiruroidea. Zeitsch. f. Parasit., VII, 273-335.

Desportes (C.), 1947. - Sur les caractères spécifiques d'une nouvelle espèce de Cucullanida, Seuratum cadarachense n. sp., de l'intestin du Lérot (Eliomys quercinus L.). Ann. Parasit., XXII, 45-52.

Dollfus (R. Ph.) et Chabaud (A. G.), 1957. - Phénomènes de convergence chez les Spirurides, en particulier dans les sous-familles Habronematine Chitwood et Wehr 1932 et Schistorophine L. Travassos 1918 ; leur importance pour une classification naturelle des Spirurides (Nematoda). Bull. Soc. Zool. France, LXXXII, 88-102.

Gendre (E.), 1926. - Sur un Nématode nouveau, parasite des Poissons. Bull. Soc. Path. Exot., XIX, 799-802.

Gendre (E.), in Joyeux (Ch.), Gendre (E.) et Baer (J.-G.), 1928. - Recherches sur les Helminthes de l'Afrique Occidentale Française. Coll. Soc. Path. Exot., Monographie II, 1-120, fig. 1-50.

GNÉDina (M.-P.), 1933, - Sur un Nématode nouveau des Oiseaux : Skrjabinura spiralis n. g., n. sp. Ann. Parasit., XI, 180-184.

HALL (M. C.), 1916. - Nematode parasites of mammals of the orders Rodentia, Lagomorpha and 'Iyracoidea. Proc. U. S. Nation. Museum, L, 1-258.

Hsü (H. F.), 1933. - On some parasitic nematodes collected in China. Parasit., XXIV, 512-541.

Inglis (W. G.), 1958. - A redescription of the Nematode Paraspidodera sellsi Morgan, 1927 and its removal to a new genus Morgascaridia. J. Helminth., XXXII, 65-72.

Inglis (W. G.) et Chabaud (A. G.), 1960. - Sur la position systématique des Schneidernematine (Nematoda). Ann. Parasit. (sous presse).

Johnston (T. H.) et Mawson (P. M.), 1940. - Some nematodes parasitic in Australian fresh-water fish. Trans. Royal Soc. South Australia, LXIV, 340-352.

Johnston (T. H.) et Mawson (P. M.), 1941 a. - Some nematodes from Australian birds of prey. Trans. Royal Soc. South Australia, LXV, 30-35.

Johnston (T. H.) et Mawson (P. M.), $1941 \mathrm{~b}$. - Some nematode parasites of Australian birds. Proc. Linnean Soc. New South Wales, LXVI, 250-256.

Johnston (T. H.) et Mawson (P. M.), $1941 \mathrm{c}$. - Some parasitic Nematodes in the collection of the Australian Museum. Rec. of the Austral. Mus., XXI, $9-16$.

LAyman (E. M.), 1933. - Ueber die parasitischen Würmer der Fische des Bailkalsees. Trav. Stat. limnol. lac Bajkal, IV, 5-99 + pl. I-VII (en russe, résumé en allemand).

Lr (H. C.), 1934. - Report on a collection of parasitic nematodes, mainly from North China. Part II. Spiruroidea. Trans. Amer. Micr. Soc., LIII, 174195 , fig. 1-43.

Moulton (J. M.), 1931. - A new species of Haplonema Ward et Magath 1916 from the stomach of Lota maculosa. J. Parasit., XVIII, 105-107 + pl. IX. 
Rallliet (A.), 1916. - Analyse du travail de Hall : « Nématodes parasites des Rongeurs ». Rec. Med. Veter., XCII, 517-521.

Rankin (J. S.), 1937. - New Helminths from North Carolina Salamanders. J. of Parasit., XXIII, 29-42.

Seurat (L. G.), 1915. - Sur un nouvel Ophiostomum parasite du Gundi. Compte Rendu Séance Soc. Biol., LXXVII, 20-22.

SKRJabiN (K. I.), Schikobalowa (N. P.) et Sobolev (A. A.), 1949. - Opredelitel' parasititcheskikh nematod: t. I, Spirurati, Filariati, 519 pp. (en russe).

Skrjabin (K. I.), Schikobalowa (N. P.), Sobolev (A. A.), Paramonov (A. A.) et Soudarikov (V. E.), 1954. - Opredelitel' parasititcheskikh nematod: t. IV, Camallanati, Rhabditati, Tylenchati, Trichocephalati, Dioctophylati, 927 pp. (en russe).

Steward (F. H.), 1914. - Studies in Indian helminthology, $\mathrm{n}^{\circ}$ 1. Rec. Indian Mus., X, 165-193 + pl. XVIII-XXIII.

Sudarikov (V. E.) et Ryjikov (K. M.), 1952. - Création d'une nouvelle famille de Nématodes parasites de Poissons d'eaux douces (Spirurata: Haplonematidx nov. fam.). Trudi Gelminth. Labor., VI, 152-157 (en russe).

Sypliaxova (O. M.), 1930. - Sur un nouveau nématode des Reptiles : Skrjabinelazia taurica n. gen., n. sp. Ann. Parasit., VIII, 615-618, fig. 1-5.

Thомas-Mawson (P. M.), 1960. - Seuratinema Johnston et Mawson 1941, synonyme de Skrjabinura Gnédina 1933. Ann. Parasit. (sous presse).

Travassos (L.), 1926. - Ascaris retusa (Rudolph, 1819). Biol., IV, 87-93.

Walton (A. C.), 1940. - Some nematodes from Tennessee amphibia. J. Tennessee Acad. Sc., XV, 402-405 (travail non consulté).

Ward (H. B.) et Magath (T. B.), 1916. - Notes on Some Nematodes from FreshWater Fishes. J. of Parasit., III, 57-64 + fig. 1-10.

Yorke (W.) et Maplestone (P. A.), 1926. - The Nematode Parasites of Vertebrates, London, 1926 , vII +536 . 